\title{
STRICT SINGULARITY OF A VOLTERRA-TYPE INTEGRAL OPERATOR ON $H^{p}$
}

\author{
SANTERI MIIHKINEN \\ (Communicated by Pamela B. Gorkin)
}

ABstract. We prove that the Volterra-type integral operator

$$
T_{g} f(z)=\int_{0}^{z} f(\zeta) g^{\prime}(\zeta) d \zeta, \quad z \in \mathbb{D},
$$

defined on the Hardy spaces $H^{p}$ fixes an isomorphic copy of $\ell^{p}$ if it is not compact. In particular, the strict singularity of $T_{g}$ coincides with its compactness on spaces $H^{p}$. As a consequence, we obtain a new proof for the equivalence of the compactness and the weak compactness of $T_{g}$ on $H^{1}$. Moreover, a non-compact $T_{g}$ acting on the space $B M O A$ fixes an isomorphic copy of $c_{0}$.

\section{INTRODUCTION}

Let $g$ be a fixed analytic function in the open unit disc $\mathbb{D}$ of the complex plane $\mathbb{C}$. We consider a linear integral operator $T_{g}$ defined for analytic functions $f$ in $\mathbb{D}$ by

$$
T_{g} f(z)=\int_{0}^{z} f(\zeta) g^{\prime}(\zeta) d \zeta, \quad z \in \mathbb{D} .
$$

Ch. Pommerenke was the first author to consider the boundedness of the operator $T_{g}$ on the Hardy space $H^{2}$ and he characterized it in connection to exponentials of $B M O A$ functions [14. A systematic study of $T_{g}$ was initiated by A. Aleman and A. G. Siskakis in [3], who showed that $T_{g}$ is bounded (compact) on the Hardy spaces $H^{p}, 1 \leq p<\infty$, if and only if $g \in B M O A(g \in V M O A)$. The same boundedness characterization of the operator $T_{g}$ on $H^{p}, 0<p<1$, spaces was obtained by Aleman and J. Cima in [2]. Here BMOA and $V M O A$ denote the spaces of analytic functions in $\mathbb{D}$ with boundary values of bounded mean oscillation and vanishing mean oscillation respectively. See also surveys [1] and [15] for more background and results on $T_{g}$.

A bounded operator $S: X \rightarrow Y$ between Banach spaces is strictly singular if its restriction to any infinite-dimensional closed subspace is not an isomorphism onto its range. This notion generalizes the concept of compact operators and it was introduced by T. Kato in [9]. Canonical examples of non-compact strictly singular operators are the inclusion mappings $i_{p, q}: \ell^{p} \hookrightarrow \ell^{q}$, where $1 \leq p<q<\infty$.

Received by the editors January 20, 2016 and, in revised form, March 1, 2016.

2010 Mathematics Subject Classification. Primary 47G10; Secondary 30H10.

Key words and phrases. Volterra operator, integral operator, strict singularity, strictly singular, Hardy spaces.

This research was supported by the Academy of Finland via the Centre of Excellence in Analysis and Dynamics Research. 
There also exist non-compact strictly singular operators on $H^{p}$ spaces for $1 \leq p<$ $\infty, p \neq 2$. To construct such an operator, one may consider cases $1 \leq p<2$ and $2<p<\infty$ separately and use the fact that $H^{p}, 1 \leq p<\infty$, contains complemented copies of $\ell^{2}$ and $\ell^{p}$; see e.g. 18 for $p=1$. In the first case, one considers a bounded projection from $H^{p}$ onto its closed subspace $M$, which is isomorphic to $\ell^{p}$. Then one utilizes the inclusion mapping $i_{p, 2}$ and the fact that $\ell^{2}$ can be embedded in $H^{p}$. In the second case, one interchanges the roles of $\ell^{p}$ and $\ell^{2}$ and repeats the reasoning above.

The aim of this note is to show that every non-compact operator $T_{g}$ acting on a Hardy space $H^{p}, 1 \leq p<\infty$, fixes an isomorphic copy of $\ell^{p}$. In particular, this implies that $T_{g}$ is strictly singular on $H^{p}$ if and only if it is compact. This article was partly motivated by [11], where the same question was studied in connection to composition operators. For the case $p=\infty$; see also [5].

Our main result is the following theorem.

Theorem 1.1. Let $g \in B M O A \backslash V M O A$ and $1 \leq p<\infty$. Then the operator $T_{g}: H^{p} \rightarrow H^{p}$ fixes an isomorphic copy of $\ell^{p}$ inside $H^{p}$, that is, there exists a subspace $M \subset H^{p}$ which is isomorphic to $\ell^{p}$ and such that the restriction of $T_{g}$ to $M$ is an isomorphism onto its range. In particular, $T_{g}$ is not strictly singular.

We should point out that there is a striking extrapolation result concerning strict singularity by Hernández, Semenov, and Tradacete in [7, Theorem 3.3]. It states that if an operator $S$ is bounded on $L_{\mathbb{R}}^{p}(E)$ and $L_{\mathbb{R}}^{q}(E)$ for some $1<p<q<\infty$ and strictly singular on $L_{\mathbb{R}}^{r}(E)$ for some $p<r<q$, then it is compact on $L_{\mathbb{R}}^{s}(E)$ for all $p<s<q$. Here $L_{\mathbb{R}}^{p}(E)$ stands for the $L^{p}$ space of real-valued functions on a finite measure space $E$. Taking the complex-valued counterpart of this result for granted, we may deduce the equivalence of strict singularity and compactness of $T_{g}$ on $H^{p}$ for $1<p<\infty$ by using the Riesz projection in the following way: Recall that strictly singular operators form a two-sided (closed) ideal in the space $\mathcal{L}\left(L^{p}\right)$ of bounded operators on $L^{p}=L_{\mathbb{C}}^{p}(\mathbb{T})$, where $\mathbb{T}=\partial \mathbb{D}$. Therefore the strict singularity of $T_{g}: H^{p} \rightarrow H^{p}$ implies that $T_{g} R: L^{p} \rightarrow L^{p}$ is strictly singular, where $R: L^{p} \rightarrow H^{p}$ is the Riesz projection and we have identified $T_{g}: H^{p} \rightarrow H^{p}$ with $T_{g}: H^{p} \rightarrow L^{p}$. Since the condition $g \in B M O A$ characterizes the boundedness of $T_{g}$ on every $H^{q}$ space and the Riesz projection is bounded on the scale $1<q<\infty$, we get that $T_{g} R$ is bounded on every $L^{q}, 1<q<\infty$, space. Now assuming that the complex version of the interpolation result is valid, it follows that $T_{g} R$ is compact on $L^{p}$ and consequently the restriction $\left.T_{g} R\right|_{H^{p}}=T_{g}$ is compact on $H^{p}$.

Theorem 1.1 however, makes a stronger statement: a non-compact operator $T_{g}$ on $H^{p}$ fixes an isomorphic copy of $\ell^{p}$. This holds for $p=1$ as well, in which case we obtain a new proof for the equivalence of the compactness and the weak compactness of $T_{g}$ on $H^{1}$ : If $g \in B M O A \backslash V M O A$, i.e., the operator $T_{g}$ is not compact, then by Theorem $1.1 T_{g}$ fixes an isomorphic copy of $\ell^{1}$ and consequently it is not weakly compact.

After giving some preliminaries in Section 2, we give the proof of Theorem 1.1 in Section 3. In Section 4, we consider the case of $T_{g}$ acting on the space BMOA and we make some remarks on strict singularity of $T_{g}$ on other spaces.

Finally, we point out that the notions of strict singularity and compactness of an operator can also be defined in a more general setting, for example in quasi-Banach spaces; see [8, Section 7.1]. Examples of such spaces are the Hardy spaces $H^{p}$ when $0<p<1$. The same compactness and boundedness characterizations of $T_{g}$ as in 
the case $1 \leq p<\infty$ also hold when $0<p<1$; see [2]. On the scale $0<p<1$, the triangle inequality is replaced by an inequality of the type $\|f+g\|_{p} \leq C\left(\|f\|_{p}+\|g\|_{p}\right)$ for some constant $C>1$ for all $f, g \in H^{p}$. This brings only some (multiplicative) constants to the proofs of results and lemmas in this article. So a non-compact $T_{g}: H^{p} \rightarrow H^{p}$ fixes an isomorphic copy of $\ell^{p}$ also in the case $0<p<1$.

\section{Preliminaries}

In this section, we briefly recall some spaces of analytic functions that appear later.

Let $H(\mathbb{D})$ be the algebra of analytic functions in $\mathbb{D}$. We define the Hardy spaces

$$
H^{p}=\left\{f \in H(\mathbb{D}):\|f\|_{p}=\left(\sup _{0 \leq r<1} \frac{1}{2 \pi} \int_{0}^{2 \pi}\left|f\left(r e^{i t}\right)\right|^{p} d t\right)^{1 / p}<\infty\right\} .
$$

The space $B M O A$ consists of functions $f \in H(\mathbb{D})$ with

$$
\|f\|_{*}=\sup _{a \in \mathbb{D}}\left\|f \circ \sigma_{a}-f(a)\right\|_{2}<\infty,
$$

where $\sigma_{a}(z)=(a-z) /(1-\bar{a} z)$ is the Möbius automorphism of $\mathbb{D}$ that interchanges the origin and the point $a \in \mathbb{D}$. Its closed subspace $V M O A$ consists of those $f \in B M O A$ with

$$
\limsup _{|a| \rightarrow 1}\left\|f \circ \sigma_{a}-f(a)\right\|_{2}=0 .
$$

Every BMOA function $f$ satisfies "a reverse Hölder's inequality", which implies that for each $0<p<\infty$ we have that

$$
\|f\|_{*} \simeq \sup _{a \in \mathbb{D}}\left\|f \circ \sigma_{a}-f(a)\right\|_{p}<\infty,
$$

where each side is bounded above by a constant multiple of the other. See e.g. 6 for more information on the spaces $B M O A$ and $V M O A$.

\section{Proof of the MAin Result}

Our goal is to show that a non-compact operator $T_{g}: H^{p} \rightarrow H^{p}, 1 \leq p<$ $\infty, g \in B M O A \backslash V M O A$, fixes an isomorphic copy of $\ell^{p}$ and hence the compactness and strict singularity are equivalent for $T_{g}$ on $H^{p}$. This is done by constructing bounded operators $V: \ell^{p} \rightarrow H^{p}$ and $U: \ell^{p} \rightarrow H^{p}$ such that the diagram in Figure 11 commutes $\left(U=T_{g} V\right)$, where $V\left(\ell^{p}\right)=M$ is the closed linear span of suitably chosen test functions $f_{a_{k}} \in H^{p}$ and the operator $U$ is an isomorphism onto its range $U\left(\ell^{p}\right)=T_{g}(M)$.

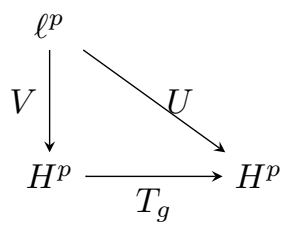

Figure 1. Operators $U, V$ and $T_{g}$ 
The strategy for choosing the suitable test functions in Propositions 3.2 and 3.5 below is similar to the one used by Laitila et al. in [11], where they utilized these test functions to show that a non-compact composition operator $C_{\varphi}: H^{p} \rightarrow H^{p}$, where $\varphi: \mathbb{D} \rightarrow \mathbb{D}$ is analytic, fixes an isomorphic copy of $\ell^{p}$. However, due to the distinct nature of operators $T_{g}$ and $C_{\varphi}$, a different kind of analysis is needed in our case.

Before proving our main result (Theorem 1.1), we provide some preparatory material. We first state a localization lemma for the standard test functions in $H^{p}, 1 \leq p<\infty$, defined by

$$
f_{a}(z)=\left[\frac{1-|a|^{2}}{(1-\bar{a} z)^{2}}\right]^{1 / p}, \quad z \in \mathbb{D},
$$

for each $a \in \mathbb{D}$. Observe that $\left\|f_{a}\right\|_{p}=1$ for all $a \in \mathbb{D}$. The proof of the lemma is straightforward and therefore omitted.

Lemma 3.1. Let $1 \leq p<\infty$ and $m$ be the normalized Lebesgue measure on $\mathbb{T}$. Define

$$
A_{\varepsilon}=\left\{e^{i \theta}:\left|e^{i \theta}-1\right|<\varepsilon\right\}
$$

for $\varepsilon>0$. Then

$$
\begin{aligned}
& \text { (i) } \lim _{a \rightarrow 1} \int_{\mathbb{T} \backslash A_{\varepsilon}}\left|f_{a}\right|^{p} d m=0 \text { for each } \varepsilon>0 . \\
& \text { (ii) } \lim _{\varepsilon \rightarrow 0} \int_{A_{\varepsilon}}\left|f_{a}\right|^{p} d m=0 \text { for each } a \in \mathbb{D} .
\end{aligned}
$$

Next, utilizing test functions $f_{a_{k}}, a_{k} \in \mathbb{D}$, for which $\left|a_{k}\right| \rightarrow 1$ sufficiently fast, we construct a certain type of bounded operator $V: \ell^{p} \rightarrow H^{p}$.

Proposition 3.2. Let $1 \leq p<\infty$ and $\left(a_{n}\right) \subset \mathbb{D}$ be a sequence such that $a_{n} \rightarrow$ $\omega \in \mathbb{T}$. Then there exists a subsequence $\left(b_{n}\right)$ of $\left(a_{n}\right)$ so that the mapping

$$
V: \ell^{p} \rightarrow H^{p}, V(\alpha)=\sum_{n=1}^{\infty} \alpha_{n} f_{b_{n}},
$$

where $\alpha=\left(\alpha_{n}\right) \in \ell^{p}$, is bounded.

Proof. We may assume that $\omega=1$. For each $\varepsilon>0$, we consider the set $A_{\varepsilon}$ defined in Lemma 3.1. Using the fact that $\left\|f_{a}\right\|_{p}=1$ for all $a \in \mathbb{D}$ and Lemma 3.1 we choose positive numbers $\varepsilon_{n}$ with $\varepsilon_{1}=2 \pi>\varepsilon_{2}>\ldots>0$ and a subsequence $\left(b_{n}\right)$ of $\left(a_{n}\right)$ such that the following conditions hold:

$$
\begin{aligned}
& \text { (i) }\left(\int_{A_{n}}\left|f_{b_{j}}\right|^{p} d m\right)^{1 / p}<4^{-n}, \quad j=1, \ldots, n-1 ; \\
& \text { (ii) }\left(\int_{\mathbb{T} \backslash A_{n}}\left|f_{b_{n}}\right|^{p} d m\right)^{1 / p}<4^{-n},
\end{aligned}
$$

for every $n \geq 1$, where $A_{n}=A_{\varepsilon_{n}}$. 
Using conditions (i)-(ii) and the fact that $\left(\int_{A_{n}}\left|f_{b_{n}}\right|^{p} d m\right)^{1 / p} \leq 1$ for all $n$, we show the upper bound $\|V \alpha\|_{p} \leq C\|\alpha\|_{\ell^{p}}$ for all $\alpha=\left(\alpha_{j}\right) \in \ell^{p}$, where $C>0$ may depend on $p$ :

$$
\begin{aligned}
\|V \alpha\|_{p}^{p} & =\int_{\mathbb{T}}\left|\sum_{j=1}^{\infty} \alpha_{j} f_{b_{j}}\right|^{p} d m=\sum_{n=1}^{\infty} \int_{A_{n} \backslash A_{n+1}}\left|\sum_{j=1}^{\infty} \alpha_{j} f_{b_{j}}\right|^{p} d m \\
& \leq \sum_{n=1}^{\infty}\left(\sum_{j=1}^{\infty}\left|\alpha_{j}\right|\left(\int_{A_{n} \backslash A_{n+1}}\left|f_{b_{j}}\right|^{p} d m\right)^{1 / p}\right)^{p} \\
& \leq \sum_{n=1}^{\infty}\left(\left|\alpha_{n}\right|\left(\int_{A_{n} \backslash A_{n+1}}\left|f_{b_{n}}\right|^{p} d m\right)^{1 / p}\right. \\
& \left.+\sum_{j \neq n}\left|\alpha_{j}\right|\left(\int_{A_{n} \backslash A_{n+1}}\left|f_{b_{j}}\right|^{p} d m\right)^{1 / p}\right)^{p},
\end{aligned}
$$

where

$$
\left(\int_{A_{n} \backslash A_{n+1}}\left|f_{b_{j}}\right|^{p} d m\right)^{1 / p} \leq\left(\int_{A_{n}}\left|f_{b_{j}}\right|^{p} d m\right)^{1 / p}<4^{-n}
$$

for $j<n$ by condition (i) and

$$
\left(\int_{A_{n} \backslash A_{n+1}}\left|f_{b_{j}}\right|^{p} d m\right)^{1 / p} \leq\left(\int_{\mathbb{T} \backslash A_{j}}\left|f_{b_{j}}\right|^{p} d m\right)^{1 / p}<4^{-j}
$$

for $j>n$ by condition (ii). Thus by estimates (2) and (3), we have that

$$
\left(\int_{A_{n} \backslash A_{n+1}}\left|f_{b_{j}}\right|^{p} d m\right)^{1 / p}<2^{-n-j}
$$

for $j \neq n$. By using estimate (4) we get

$$
\begin{aligned}
\|V(\alpha)\|_{p}^{p} & \leq \sum_{n=1}^{\infty}\left(\left|\alpha_{n}\right|\left(\int_{A_{n} \backslash A_{n+1}}\left|f_{b_{n}}\right|^{p} d m\right)^{1 / p}+\sum_{j \neq n}\left|\alpha_{j}\right| 2^{-n-j}\right)^{p} \\
& \leq \sum_{n=1}^{\infty}\left(\left|\alpha_{n}\right|+\|\alpha\|_{\ell^{p}} 2^{-n}\right)^{p} \\
& \leq 2^{p}\left(\sum_{n=1}^{\infty}\left|\alpha_{n}\right|^{p}+\|\alpha\|_{\ell^{p}}^{p} \sum_{n=1}^{\infty} 2^{-n p}\right) \leq 2^{p+1}\|\alpha\|_{\ell^{p}}^{p} .
\end{aligned}
$$

The next result is an observation by Aleman and Cima [2] and it states that for a non-compact operator $T_{g}$ on $H^{p}$ we can find a sequence $\left(f_{a_{k}}\right)$ of test functions so that the sequence $\left(\left\|T_{g} f_{a_{k}}\right\|_{p}\right)$ converges to a positive constant. Its proof is based on the fact that for all $0<p<\infty$ and $t \in(0, p / 2)$ there exists a constant $C=C(p, t)>0$ such that

$$
\left\|T_{g} f_{a}\right\|_{p} \geq C\left\|g \circ \sigma_{a}-g(a)\right\|_{t}
$$

for all $a \in \mathbb{D}$, where $\sigma_{a}(z)=(a-z) /(1-\bar{a} z)$; see [2, Theorem 3]. 
Proposition 3.3. Let $g \in B M O A \backslash V M O A$ and $1 \leq p<\infty$. Then

$$
c:=\limsup _{|a| \rightarrow 1}\left\|T_{g} f_{a}\right\|_{p}>0 .
$$

In particular, there exists a sequence $\left(a_{k}\right) \subset \mathbb{D}, a_{k} \rightarrow \omega \in \mathbb{T}$ so that

$$
\lim _{k \rightarrow \infty}\left\|T_{g} f_{a_{k}}\right\|_{p}=c .
$$

Next, we prove a localization result for the images $T_{g} f_{a}$ of the test functions $f_{a}$ (cf. Lemma 3.1).

Lemma 3.4. Let $g \in B M O A, 1 \leq p<\infty$, and $\left(a_{k}\right) \subset \mathbb{D}$ be a sequence such that $a_{k} \rightarrow \omega \in \mathbb{T}$. Define

$$
A_{\varepsilon}=\left\{e^{i \theta}:\left|e^{i \theta}-\omega\right|<\varepsilon\right\}
$$

for each $\varepsilon>0$. Then

$$
\begin{aligned}
& \text { (i) } \lim _{k \rightarrow \infty} \int_{\mathbb{T} \backslash A_{\varepsilon}}\left|T_{g} f_{a_{k}}\right|^{p} d m=0 \text { for every } \varepsilon>0 . \\
& \text { (ii) } \lim _{\varepsilon \rightarrow 0} \int_{A_{\varepsilon}}\left|T_{g} f_{a_{k}}\right|^{p} d m=0 \text { for each } k .
\end{aligned}
$$

Proof. (i) Let $\varepsilon>0$. For the simplicity of notation, we may assume that $\omega=1$. Also, we assume that $g(0)=0$. We have that

$$
\left|1-\overline{a_{k}} s e^{i \theta}\right| \geq \delta
$$

for all $k, 0 \leq s \leq 1$, and $\varepsilon \leq|\theta| \leq \pi$, where $\delta>0$. Thus for those $s$ and $\theta$ we have

$$
\left|f_{a_{k}}\left(s e^{i \theta}\right)\right|^{p}=\frac{1-\left|a_{k}\right|^{2}}{\left|1-\overline{a_{k}} s e^{i \theta}\right|^{2}} \leq \frac{1-\left|a_{k}\right|^{2}}{\delta^{2}}
$$

and

$$
\left|f_{a_{k}}^{\prime}\left(s e^{i \theta}\right)\right|^{p} \leq C \frac{1-\left|a_{k}\right|^{2}}{\left|1-\overline{a_{k}} s e^{i \theta}\right|^{2+p}} \leq C \frac{1-\left|a_{k}\right|^{2}}{\delta^{2+p}}
$$

for all $k$, where $C>0$ may depend on $p$. For $\zeta \in \mathbb{T} \backslash A_{\varepsilon}$, we obtain

$$
\begin{aligned}
\left|T_{g} f_{a_{k}}(\zeta)\right|^{p} & =\left|\int_{0}^{1} f_{a_{k}}(s \zeta) g^{\prime}(s \zeta) \zeta d s\right|^{p} \\
& \leq C\left(\left|f_{a_{k}}(\zeta) g(\zeta)\right|^{p}+\left(\int_{0}^{1}\left|f_{a_{k}}^{\prime}(s \zeta) g(s \zeta)\right| d s\right)^{p}\right) \\
& \leq C\left(\frac{1-\left|a_{k}\right|}{\delta^{2}}|g(\zeta)|^{p}+\frac{1-\left|a_{k}\right|}{\delta^{2+p}}\left(\int_{0}^{1}|g(s \zeta)| d s\right)^{p}\right)
\end{aligned}
$$

where constants $C>0$ may depend on $p$ and change from one instance to another. Since $g \in B M O A$, we have that $|g(z)| \leq C \log \left(\frac{1}{1-|z|}\right)\|g\|_{*}$ for some $C>0$, and consequently $\int_{0}^{1}|g(s \zeta)| d s \leq C\|g\|_{*}$. Therefore

$$
\int_{\mathbb{T} \backslash A_{\varepsilon}}\left|T_{g} f_{a_{k}}\right|^{p} d m \leq C\left(\frac{1-\left|a_{k}\right|}{\delta^{2}}\|g\|_{p}^{p}+\frac{1-\left|a_{k}\right|}{\delta^{2+p}}\|g\|_{*}^{p}\right) \rightarrow 0
$$

as $k \rightarrow \infty$, where $\|g\|_{p} \leq \sup _{b \in \mathbb{D}}\left\|g \circ \sigma_{b}-g(b)\right\|_{p} \simeq\|g\|_{*}<\infty$; see (11). 
(ii) If $k$ is fixed, then it follows from the absolute continuity of the measure $B \mapsto \int_{B}\left|T_{g} f_{a_{k}}\right|^{p} d m$ that $\int_{A_{\varepsilon}}\left|T_{g} f_{a_{k}}\right|^{p} d m \rightarrow 0$ as $\varepsilon \rightarrow 0$.

As a final step towards the proof of Theorem 1.1 we construct an isomorphism from $\ell^{p}$ into $H^{p}$ using a non-compact $T_{g}$ and test functions.

Proposition 3.5. Let $g \in B M O A \backslash V M O A, 1 \leq p<\infty$, and $\left(a_{n}\right) \subset \mathbb{D}$ be the sequence from Proposition 3.3. Then there exists a subsequence $\left(b_{n}\right)$ of $\left(a_{n}\right)$ such that the mapping

$$
U: \ell^{p} \rightarrow H^{p}, U(\alpha)=\sum_{n=1}^{\infty} \alpha_{n} T_{g} f_{b_{n}}
$$

where $\alpha=\left(\alpha_{n}\right) \in \ell^{p}$, is an isomorphism onto its range.

Proof. By Proposition 3.2 there exists a subsequence $\left(c_{n}\right)$ of $\left(a_{n}\right)$ inducing a bounded operator

$$
S: \ell^{p} \rightarrow H^{p}, S(\alpha)=\sum_{n=1}^{\infty} \alpha_{n} f_{c_{n}},
$$

and for any subsequence $\left(b_{n}\right)$ of $\left(c_{n}\right)$ the operator $V: \ell^{p} \rightarrow H^{p}, V(\alpha)=\sum_{n=1}^{\infty} \alpha_{n} f_{b_{n}}$ is bounded. After finding the suitable sequence $\left(b_{n}\right)$, the operator $U=T_{g} V$ will be bounded as a composition of two bounded operators.

Before proving that $U$ is bounded from below, we provide some preparatory material. Since $\left(c_{n}\right)$ is a subsequence of $\left(a_{n}\right)$, we have that $c_{n} \rightarrow \omega \in \mathbb{T}$ and there exists a number $c>0$ such that

$$
\lim _{n \rightarrow \infty}\left\|T_{g} f_{c_{n}}\right\|_{p}=c
$$

by Proposition 3.3. Using Proposition 3.3 and Lemma 3.4 we choose positive numbers $\varepsilon_{n}$ with $\varepsilon_{1}>\varepsilon_{2}>\ldots>0$ and a subsequence $\left(b_{n}\right)$ of $\left(c_{n}\right)$ such that the following conditions hold:

$$
\begin{aligned}
\text { (i) } & \left(\int_{A_{n}}\left|T_{g} f_{b_{j}}\right|^{p} d m\right)^{1 / p}<4^{-n} \delta c, \quad j=1, \ldots, n-1, \\
\text { (ii) } & \left(\int_{\mathbb{T} \backslash A_{n}}\left|T_{g} f_{b_{n}}\right|^{p} d m\right)^{1 / p}<4^{-n} \delta c, \\
\text { (iii) } & \frac{c}{2} \leq\left(\int_{A_{n}}\left|T_{g} f_{b_{n}}\right|^{p} d m\right)^{1 / p} \leq 2 c,
\end{aligned}
$$

for every $n \geq 1$, where

$$
A_{n}=A_{\varepsilon_{n}}=\left\{e^{i \theta}:\left|e^{i \theta}-\omega\right|<\varepsilon_{n}\right\}
$$

and $\delta>0$ is a constant whose value is determined later. 
Now we are ready to prove that $U$ is bounded from below. Using conditions (ii) and (iii), we get

$$
\begin{aligned}
\|U \alpha\|_{p}^{p}= & \int_{\mathbb{T}}\left|\sum_{j=1}^{\infty} \alpha_{j} T_{g} f_{b_{j}}\right|^{p} d m \geq \sum_{n=1}^{\infty} \int_{A_{n} \backslash A_{n+1}}\left|\sum_{j=1}^{\infty} \alpha_{j} T_{g} f_{b_{j}}\right|^{p} d m \\
\geq & \sum_{n=1}^{\infty}|| \alpha_{n} \mid\left(\int_{A_{n} \backslash A_{n+1}}\left|T_{g} f_{b_{n}}\right|^{p} d m\right)^{1 / p} \\
& -\left.\left.\left(\int_{A_{n} \backslash A_{n+1}}\left|\sum_{j \neq n} \alpha_{j} T_{g} f_{b_{j}}\right|^{p} d m\right)^{1 / p}\right|^{p}\right|_{n=1} ^{\infty}\left(2^{-p}\left|\alpha_{n}\right|^{p}\left(\frac{c}{2}-4^{-n-1} \delta c\right)^{p}-\int_{A_{n} \backslash A_{n+1}}\left|\sum_{j \neq n} \alpha_{j} T_{g} f_{b_{j}}\right|^{p} d m\right) \\
\geq & \sum_{n=1}^{\infty}\left(2^{-p}\left|\alpha_{n}\right|^{p}\left(\frac{c}{2}-4^{-n-1} \delta c\right)^{p}\right. \\
& \left.\quad\left(\sum_{j \neq n}\left|\alpha_{j}\right|\left(\int_{A_{n} \backslash A_{n+1}}\left|T_{g} f_{b_{j}}\right|^{p} d m\right)^{1 / p}\right)^{p}\right),
\end{aligned}
$$

where

$$
\left(\int_{A_{n} \backslash A_{n+1}}\left|T_{g} f_{b_{j}}\right|^{p} d m\right)^{1 / p} \leq\left(\int_{A_{n}}\left|T_{g} f_{b_{j}}\right|^{p} d m\right)^{1 / p}<4^{-n} \delta c
$$

for $j<n$ by condition (i) and

$$
\left(\int_{A_{n} \backslash A_{n+1}}\left|T_{g} f_{b_{j}}\right|^{p} d m\right)^{1 / p} \leq\left(\int_{\mathbb{T} \backslash A_{j}}\left|T_{g} f_{b_{j}}\right|^{p} d m\right)^{1 / p}<4^{-j} \delta c
$$

for $j>n$ by condition (ii). Thus we have that

$$
\left(\int_{A_{n} \backslash A_{n+1}}\left|T_{g} f_{b_{j}}\right|^{p} d m\right)^{1 / p}<2^{-n-j} \delta c
$$


for $j \neq n$. Consequently, we can estimate

$$
\begin{aligned}
\|U \alpha\|_{p}^{p} & \geq \sum_{n=1}^{\infty}\left(2^{-p}\left|\alpha_{n}\right|^{p}\left(\frac{c}{2}-4^{-n-1} \delta c\right)^{p}-\left(\sum_{j=1}^{\infty}\left|\alpha_{j}\right| 2^{-n-j} \delta c\right)^{p}\right) \\
& \geq \sum_{n=1}^{\infty}\left(2^{-p}\left|\alpha_{n}\right|^{p}\left(\frac{c}{2}-4^{-n-1} \delta c\right)^{p}-2^{-n} \delta^{p} c^{p}\|\alpha\|_{\ell^{p}}^{p}\right) \\
& \geq 2^{-p} \sum_{n=1}^{\infty}\left|\alpha_{n}\right|^{p}\left(\frac{c}{2}-\frac{1}{16} \delta c\right)^{p}-\delta^{p} c^{p}\|\alpha\|_{\ell^{p}}^{p} \\
& \geq 2^{-p}\left(\frac{7}{16}\right)^{p} c^{p}\|\alpha\|_{\ell^{p}}^{p}-\delta^{p} c^{p}\|\alpha\|_{\ell^{p}}^{p} \\
& =\left(\left(\frac{7}{32}\right)^{p}-\delta^{p}\right) c^{p}\|\alpha\|_{\ell^{p}}^{p}=\left(\frac{1}{8}\right)^{p} c^{p}\|\alpha\|_{\ell^{p}}^{p}
\end{aligned}
$$

when we choose $0<\delta<1$ such that $\delta^{p}=\left(\frac{7}{32}\right)^{p}-\left(\frac{1}{8}\right)^{p}$, i.e., $\delta=\frac{\left(7^{p}-4^{p}\right)^{1 / p}}{32}$. Thus the bounded operator $U$ is also bounded from below and consequently it is an isomorphism onto its range.

Now our main result follows.

Proof of Theorem 1.1, By Propositions 3.2 and 3.5. we can choose a sequence $\left(b_{n}\right) \subset \mathbb{D}$ that induces a bounded operator

$$
V: \ell^{p} \rightarrow H^{p}, V(\alpha)=\sum_{n=1}^{\infty} \alpha_{n} f_{b_{n}},
$$

where $\alpha=\left(\alpha_{n}\right) \in \ell^{p}$, and an isomorphism $U: \ell^{p} \rightarrow H^{p}, U=T_{g} V$ onto its range.

Define $M=\overline{\operatorname{span}\left\{f_{b_{n}}\right\}}$, where the closure is taken in $H^{p}$. Since $U$ is bounded from below, we have that the restriction $\left.T_{g}\right|_{M}$ is bounded from below. Thus $\left.T_{g}\right|_{M}: M \rightarrow$ $T_{g}(M)$ is an isomorphism and consequently $M$ is isomorphic to $\ell^{p}$. In particular, the operator $T_{g}$ is not strictly singular.

We conclude this section by posing two questions concerning the $\ell^{2}-$ singularity of $T_{g}$. To be specific, define $S\left(H^{p}\right)$ to be the class of strictly singular operators on $H^{p}$ and $S_{r}\left(H^{p}\right)$ to be the class of $\ell^{r}$-singular operators on $H^{p}$, i.e., those bounded operators which do not fix a copy of the space $\ell^{r}$. Then $S\left(H^{p}\right)=S_{2}\left(H^{p}\right) \cap S_{p}\left(H^{p}\right)$ for $1<p<\infty$; see [17]. For a non-compact operator $T_{g}$ on $H^{p}$ it follows from Theorem 1.1 that $T_{g} \notin S_{p}\left(H^{p}\right)$. However, we did not pursue the following questions.

Questions. Does a non-compact $T_{g}: H^{p} \rightarrow H^{p}$ satisfy $T_{g} \notin S_{2}\left(H^{p}\right)$ ? Can we characterize those $g$ such that $T_{g} \in S_{2}\left(H^{p}\right)$, where $1 \leq p<\infty, p \neq 2$ ?

\section{Strict Singularity On BMOA AND Other SPACES}

In this section, we consider the strict singularity of $T_{g}$ on $B M O A$, the Bergman spaces $A^{p}, 1 \leq p<\infty$, and the Bloch space $\mathcal{B}$. The case of $B M O A$ essentially follows from the reasoning done in [10, where we utilize an idea of Leibov [12] that there exist isomorphic copies of the space $c_{0}$ of null sequences inside $V M O A$. This fact will imply that the strict singularity of $T_{g}$ on $B M O A$ or on $V M O A$ is equivalent to the compactness of $T_{g}$ on the same space. The sketch of the proof is the following. 
We recall that the boundedness of $T_{g}$ on $B M O A$ is characterized by the condition $g \in L M O A$, where $L M O A$ is the "logarithmic $B M O A$ " space; see [16].

By the proof of Theorem 2 and Lemma 6 in [10] we can find a sequence $\left(h_{n}\right)$ in $V M O A$ which is equivalent to the standard basis of $c_{0}$. If $T_{g}$ is non-compact on $V M O A$, it follows from the estimate (4.6) in [10] that $\left\|T_{g} h_{n}\right\|_{*}>c>0$ for some constant $c$ for all $n$. Since $g \in L M O A \subset B M O A$, the operator $T_{g}$ is bounded on $H^{2}$ and consequently $\left\|T_{g} h_{n}\right\|_{2} \rightarrow 0$, as $n \rightarrow \infty$. Now we apply Lemma 6 in [10] again to obtain (by passing to a subsequence, if needed) that $\left(T_{g} h_{n}\right)$ is equivalent to the standard basis of $c_{0}$. Hence $\left.T_{g}\right|_{M}$, where $M=\overline{\operatorname{span}\left\{h_{n}\right\}}$, is an isomorphism onto its range and $T_{g}$ is not strictly singular on $V M O A$ (or on $B M O A$ ).

In the Bergman spaces, boundedness and compactness of $T_{g}$ was characterized in [4. It is known that $A^{p}, 1 \leq p<\infty$, are isomorphic to $\ell^{p}$; see e.g. 19. Chapter 3.A, Theorem 11]. Hence the strict singularity of the operator $T_{g}$ coincides with the compactness, since all strictly singular operators on $\ell^{p}$ are compact; see 13 , Proposition 2.c.3] and a comment thereafter. The boundedness and compactness of $T_{g}$ acting on Bloch spaces was investigated in [20]. In this case, we can deduce as follows: If $T_{g}$ acting on $\mathcal{B}$ is strictly singular, then its restriction to the little Bloch space $\mathcal{B}_{0}$ is also strictly singular. Since $\mathcal{B}_{0}$ is isomorphic to $c_{0}$ and strictly singular operators on $c_{0}$ are compact, the restriction $\left.T_{g}\right|_{\mathcal{B}_{0}}$ is compact. Also, the bidual $\left(\mathcal{B}_{0}\right)^{* *}$ can be identified with $\mathcal{B}$, so the operator $T_{g}$ can be identified with the biadjoint operator $\left(\left.T_{g}\right|_{\mathcal{B}_{0}}\right)^{* *}$. Therefore $T_{g}$ is compact.

\section{ACKNOWLEDGEMENTS}

The author would like to thank his advisor, Pekka Nieminen, as well as HansOlav Tylli and Eero Saksman for useful discussions regarding this topic. The author also thanks the referee for the helpful comments to improve the presentation.

\section{REFERENCES}

[1] Alexandru Aleman, A class of integral operators on spaces of analytic functions, Topics in complex analysis and operator theory, Univ. Málaga, Málaga, 2007, pp. 3-30. MR2394654

[2] Alexandru Aleman and Joseph A. Cima, An integral operator on $H^{p}$ and Hardy's inequality, J. Anal. Math. 85 (2001), 157-176, DOI 10.1007/BF02788078. MR.1869606

[3] Alexandru Aleman and Aristomenis G. Siskakis, An integral operator on $H^{p}$, Complex Variables Theory Appl. 28 (1995), no. 2, 149-158. MR1700079

[4] Alexandru Aleman and Aristomenis G. Siskakis, Integration operators on Bergman spaces, Indiana Univ. Math. J. 46 (1997), no. 2, 337-356, DOI 10.1512/iumj.1997.46.1373. MR1481594

[5] José Bonet, Paweł Domański, and Mikael Lindström, Essential norm and weak compactness of composition operators on weighted Banach spaces of analytic functions, Canad. Math. Bull. 42 (1999), no. 2, 139-148, DOI 10.4153/CMB-1999-016-x. MR.1692002

[6] Daniel Girela, Analytic functions of bounded mean oscillation, Complex function spaces (Mekrijärvi, 1999), Univ. Joensuu Dept. Math. Rep. Ser., vol. 4, Univ. Joensuu, Joensuu, 2001, pp. 61-170. MR 1820090

[7] Francisco L. Hernández, Evgeny M. Semenov, and Pedro Tradacete, Strictly singular operators on $L_{p}$ spaces and interpolation, Proc. Amer. Math. Soc. 138 (2010), no. 2, 675-686, DOI 10.1090/S0002-9939-09-10089-8. MR2557184

[8] N. J. Kalton, N. T. Peck, and James W. Roberts, An F-space sampler, London Mathematical Society Lecture Note Series, vol. 89, Cambridge University Press, Cambridge, 1984. MR 808777

[9] Tosio Kato, Perturbation theory for nullity, deficiency and other quantities of linear operators, J. Analyse Math. 6 (1958), 261-322. MR0107819 
[10] Jussi Laitila, Santeri Miihkinen, and Pekka J. Nieminen, Essential norms and weak compactness of integration operators, Arch. Math. (Basel) 97 (2011), no. 1, 39-48, DOI 10.1007/s00013-011-0272-z. MR2820586

[11] J. Laitila, P. J. Nieminen, E. Saksman, and H.-O. Tylli, Structural rigidity of composition operators on $H^{p}$, (forthcoming).

[12] M. V. Lerbov, Subspaces of the space VMO (Russian), Teor. Funktsiü Funktsional. Anal. i Prilozhen. 46 (1986), 51-54, DOI 10.1007/BF01095622; English transl., J. Soviet Math. 48 (1990), no. 5, 536-538. MR 865789

[13] Joram Lindenstrauss and Lior Tzafriri, Classical Banach spaces. I, Springer-Verlag, BerlinNew York, 1977. Sequence spaces; Ergebnisse der Mathematik und ihrer Grenzgebiete, Vol. 92. MR0500056

[14] Ch. Pommerenke, Schlichte Funktionen und analytische Funktionen von beschränkter mittlerer Oszillation (German), Comment. Math. Helv. 52 (1977), no. 4, 591-602. MR0454017

[15] Aristomenis G. Siskakis, Volterra operators on spaces of analytic functions-a survey, Proceedings of the First Advanced Course in Operator Theory and Complex Analysis, Univ. Sevilla Secr. Publ., Seville, 2006, pp. 51-68. MR.2290748

[16] Aristomenis G. Siskakis and Ruhan Zhao, A Volterra type operator on spaces of analytic functions, Function spaces (Edwardsville, IL, 1998), Contemp. Math., vol. 232, Amer. Math. Soc., Providence, RI, 1999, pp. 299-311, DOI 10.1090/conm/232/03406. MR1678342

[17] L. Weis, On perturbations of Fredholm operators in $L_{p}(\mu)$-spaces, Proc. Amer. Math. Soc. 67 (1977), no. 2, 287-292. MR0467377

[18] P. Wojtaszczyk, The Banach space $H_{1}$, Functional analysis: surveys and recent results, III (Paderborn, 1983), North-Holland Math. Stud., vol. 90, North-Holland, Amsterdam, 1984, pp. 1-33, DOI 10.1016/S0304-0208(08)71464-6. MR761370

[19] P. Wojtaszczyk, Banach spaces for analysts, Cambridge Studies in Advanced Mathematics, vol. 25, Cambridge University Press, Cambridge, 1991. MR 1144277

[20] Rikio Yoneda, Integration operators on weighted Bloch spaces, Nihonkai Math. J. 12 (2001), no. 2, 123-133. MR.1869743

Department of Mathematics and Statistics, University of Helsinki, Box 68, 00014 HELSINKI, FINLAND

E-mail address: santeri.miihkinen@helsinki.fi 